\title{
Carriers of the mid-IR emission bands in PNe reanalysed
}

\section{Evidence of a link between circumstellar and interstellar aromatic dust ${ }^{\star}, \star \star$}

\author{
C. Joblin ${ }^{1}$, R. Szczerba ${ }^{2}$, O. Berné ${ }^{1}$, and C. Szyszka ${ }^{2,3}$ \\ ${ }^{1}$ Centre d'Étude Spatiale des Rayonnements, Université de Toulouse et CNRS, Observatoire Midi-Pyrénées, 9 Av. du Colonel Roche, \\ 31028 Toulouse Cedex 04, France \\ e-mail: christine.joblin@cesr.fr \\ 2 N. Copernicus Astronomical Center, Rabianska 8, 87-100 Toruń, Poland \\ 3 Nicolaus Copernicus University, Gagarina 11, 87-100 Toruń, Poland
}

Received 13 November 2007 / Accepted 4 August 2008

ABSTRACT

\begin{abstract}
Context. It has been shown that the diversity of the aromatic emission features can be rationalized into different classes of objects, in which differences between circumstellar and interstellar matter are emphasised.

Aims. We probe the links between the mid-IR emitters observed in planetary nebulae ( $\mathrm{PNe}$ ) and their counterparts in the interstellar medium in order to probe a scenario in which the latter have been formed in the circumstellar environment of evolved stars.

Methods. The mid-IR (6-14 $\mu \mathrm{m})$ emission spectra of PNe and compact H II regions were analysed on the basis of previous work on photodissociation regions (PDRs). Galactic, Large Magellanic Cloud (LMC), and Small Magellanic Cloud (SMC) objects were considered in our sample.

Results. We show that the mid-IR emission of PNe can be decomposed as the sum of six components. Some components made of polycyclic aromatic hydrocarbon (PAH) and very small grain (VSG) populations are similar to those observed in PDRs. Others are fitted in an evolutionary scenario involving the destruction of the aliphatic component observed in the post-AGB stage, as well as strong processing of PAHs in the extreme conditions of PNe that leads to a population of very large ionized PAHs. This species called $\mathrm{PAH}^{x}$ are proposed as the carriers of a characteristic band at $7.90 \mu \mathrm{m}$. This band can be used as part of diagnostics that identify PNe in nearby galaxies and is also observed in galactic compact $\mathrm{H}$ II regions.

Conclusions. These results support the formation of the aromatic very small dust particles in the envelopes of evolved stars, in the Milky Way, as well as in the LMC and SMC, and their subsequent survival in the interstellar medium.
\end{abstract}

Key words. astrochemistry - stars: carbon - ISM: planetray nebulae: general - infrared: ISM - methods: data analysis

\section{Introduction}

Evolved stars are known to play an important role in the dust formation in galaxies, but the link between circumstellar and interstellar dust is still an open question. Dust populations include the carriers of the so-called aromatic infrared bands (AIBs), which are located between 3.3 and $14 \mu \mathrm{m}$ and attributed to very small aromatic dust particles amongst which are large polycyclic aromatic hydrocarbons (PAHs). Emission in the AIBs arises following stochastic heating of their carriers by UV photons, and it is therefore often used to probe UV-excited environments in our Galaxy as well as in external galaxies (Uchida et al. 2000; Peeters et al. 2004; Brandl et al. 2006; Spoon et al. 2007; Smith et al. 2007; Lebouteiller et al. 2007). A detailed analysis of the band positions and profiles can also be used as a diagnostics of the type of emitting region (Peeters et al. 2002; van Diedenhoven et al. 2004). Ultimately, one would like to use these bands to

\footnotetext{
* This work is based on observations made with the Spitzer Space Telescope, which is operated by the Jet Propulsion Laboratory, California Institute of Technology under a contract with NASA. Based on observations with ISO, an ESA project with instruments funded by ESA Member States (especially the PI countries: France, Germany, the Netherlands and the United Kingdom) and with the participation of ISAS and NASA.

$\star \star$ Tables A.1 and A.2 are only available in electronic form at http://www . aanda.org
}

trace the chemical evolution of their carriers induced by the local physical conditions.

Several authors have tried to provide a chemical scheme based on soot formation models to form PAHs in evolved carbon stars (Frenklach \& Feigelson 1989; Cherchneff et al. 1992). This scheme, which involves the pyrolysis of hydrocarbons and more especially of $\mathrm{C}_{2} \mathrm{H}_{2}$ has gained support with the detection of $\mathrm{C}_{4} \mathrm{H}_{2}, \mathrm{C}_{6} \mathrm{H}_{2}$ and $\mathrm{C}_{6} \mathrm{H}_{6}$ in CRL618 (Cernicharo et al. 2001). The study of the AIB carriers in these cool-star environments is difficult, however, due to the few UV photons available to trigger the AIB emission. There is therefore a natural bias towards their study in planetary nebulae $(\mathrm{PNe})$ where the central star is very hot. However, the AIB spectrum in $\mathrm{PNe}$ is known to present significant differences compared to the bands observed in PDRs. In particular, the dominant band of " $7.7 \mu \mathrm{m}$ " feature arises at $7.8 \mu \mathrm{m}$ in PNe compared to $7.6 \mu \mathrm{m}$ in the ISM (Bregman 1989; Cohen et al. 1989; Peeters et al. 2002). We might therefore suspect a significant chemical evolution between PAHs in these circumstellar environments and those dominating the emission at the scale of a galaxy and located in PDRs (Hony et al. 2001). To progress on this question, we propose to analyse the spectra of several PNe in the Milky Way (MW) and in the Large and Small Magellanic Clouds (LMC and SMC) as examples of galaxies with metallicities that differ by a factor of 2 to 4 .

The analysis of the spectra is inspired from previous work by Rapacioli et al. (2005) and Berné et al. (2007) on cool PDRs. 

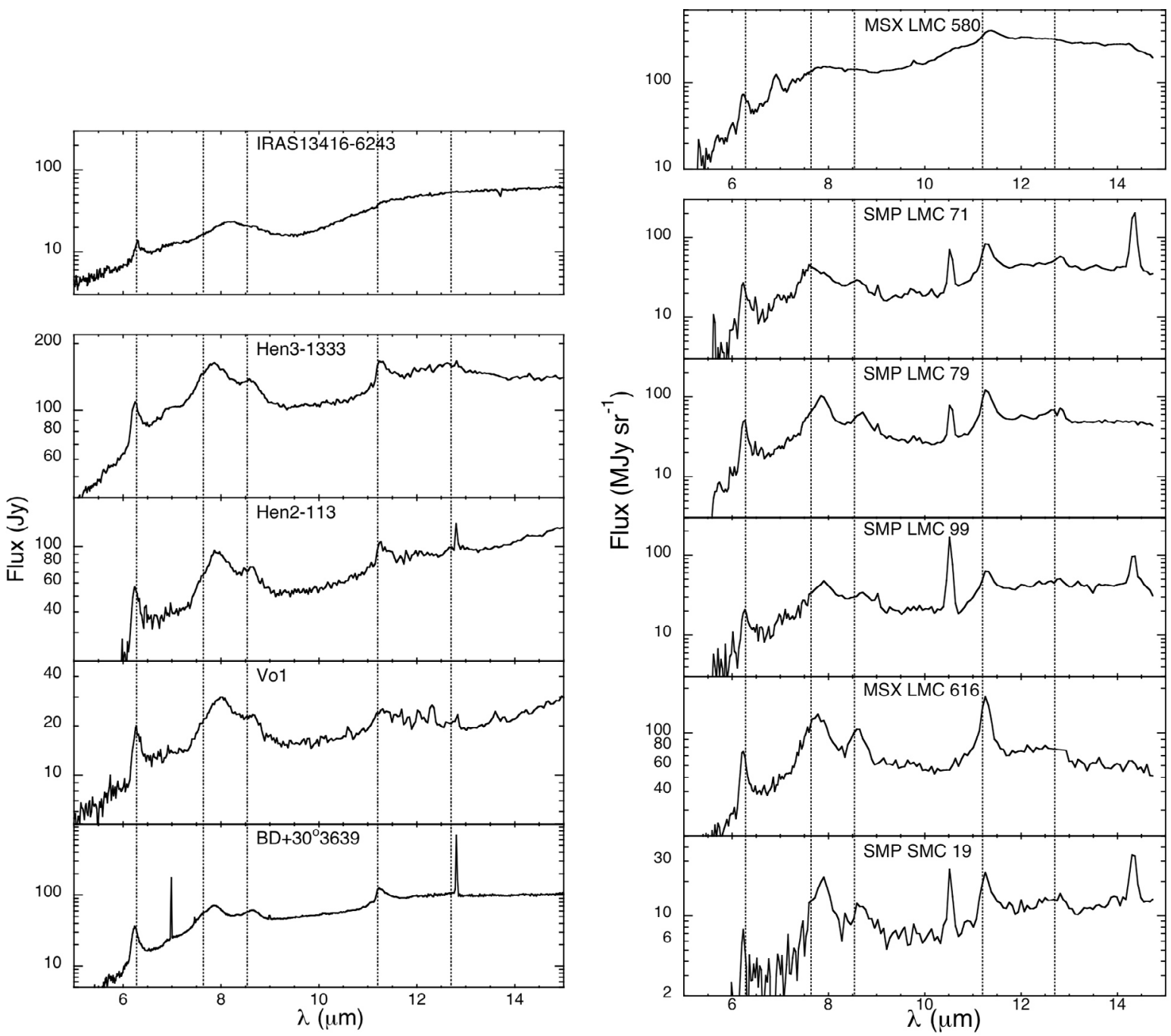

Fig. 1. Mid-IR (5-15 $\mu \mathrm{m})$ spectra of a post-AGB object (top panel) and of the studied PNe, in the Milky Way (left panel; ISO SWS01 observations) and in the LMC/SMC (right panel; Spitzer-IRS observations). Vertical lines have been drawn at 6.28, 7.64, 8.55, 11.20, $12.70 \mu \mathrm{m}$, at the peaks of the template $\mathrm{PAH}^{+}$spectrum (cf. Table A.1).

The authors find that the $7.6 \mu \mathrm{m}$ component is carried by molecular PAHs with two charge states, neutrals and cations, whereas some very small grains (VSGs) carry the mid-IR continuum and broader bands, in particular one at $\sim 7.8 \mu \mathrm{m}$. In Sect. 2 , we describe the sample of objects and data reduction. The fitting procedure is detailed in Sect. 3 and the results are presented. The interpretation follows in Sect. 4 with special emphasis on the processing of the carriers of the aromatic features in the extreme irradiation environment of PNe. This interpretation is supported by the analysis of the spectra of compact H II regions in which the hardness of the radiation field is quite comparable to that of the studied young PNe. Conclusions and implications of this work for the link between circumstellar and interstellar PAHs are given in Sect. 5.

\section{Observations and data reduction}

Figure 1 presents the $5-15 \mu \mathrm{m}$ spectra of the studied PNe in the Milky Way (left panels) and in the LMC and SMC (right panels). Spectra of two post-AGB objects are shown in the top (separate) panels just for comparison with the spectra of PNe. IRAS
13416-6243 is a C-rich post-AGB object that has been classified by Peeters et al. (2002) as the prototype object for class $\mathrm{C}$ bands. Its spectrum exhibits the $6.9 \mu \mathrm{m}$ feature that is seen only in the post-AGB phase (Hrivnak et al. 2000). IRAS 13416 does not show the double-peaked spectral energy distribution that is typical in post-AGB (cf. http: //www .ncac . torun.pl/postagb; Szczerba et al. 2007), which means that there is still strong mass loss going on or that it has been stopped very recently; as a consequence, the inner radius of the envelope is still close to the central star. MSX LMC 580 appears to have the same characteristics as IRAS 13416 with a strong $6.9 \mu \mathrm{m}$ feature, in particular. We therefore classify it as a genuine post-AGB object in LMC.

The ISO SWS01 spectra of the galactic post-AGB object IRAS 13416-6243 and galactic PNe (Hen 3-1333, Hen 2-113, PN Vo 1, and BD+30 3639) were reduced using ISAP (Szczerba et al. 1997, 2001), while the Spitzer Space Telescope (SST) low-resolution InfraRed Spectrograph (IRS) spectra (MSX LMC 580 and MSX LMC 616 - acquired from programme \#3591; SMP LMC 71, SMP LMC 79, SMP LMC 99, and SMP SMC 19 - acquired from programme \#20443) were reduced using the CUBISM software (Smith et al. 2007) without the slit-loss 
correction function, in a $4 \times 2$ pixel aperture. Background subtraction was achieved using an off-source spectrum in the same aperture. The above SMP objects have a well-established evolutionary status as being planetary nebulae (Stanghellini et al. 2007), whereas MSX LMC 616 is the newly discovered planetary nebula in the LMC (Reid \& Parker 2006).

For the discussion of the link between aromatic dust in circumstellar and interstellar environments, we have also included compact H II regions in the MW and LMC. For the MW, IRAS 18317-0757, IRAS 18502+0051, IRAS 19442+2427, IRAS $22308+5812$, and IRAS $23133+6050$ were selected as they have well-described AIB features (Peeters et al. 2002). The ISO SWS01 spectra of the selected galactic compact H II regions were reduced using ISAP and are presented in the left panels in Fig. 5. For the LMC H II regions we have selected four sources from the SST programme \#3591 (MSX LMC 1121, MSX LMC 1207, MSX LMC 1217, and MSX LMC 1798), and reduced spectroscopic data using the CUBISM software. We have checked that these sources are not known or newly discovered PNe in LMC (Reid \& Parker 2006), so the presence of gas emission lines in their IRS spectra allows us to assume that they are genuine HII regions. Their mid-IR spectra, after processing as described in Sect. 3.2 are presented in the right panels in Fig. 5.

\section{Analysis of spectra using a PAH/VSG approach}

\subsection{The basis}

Using a mathematical decomposition, Rapacioli et al. (2005) and Berné et al. (2007) were able to extract in PDRs the emission spectra of $\mathrm{PAH}$ neutrals $\left(\mathrm{PAH}^{0}\right)$ and cations $\left(\mathrm{PAH}^{+}\right)$, as well as a population of carbonaceous VSGs. The analysis of the midIR $(6-14 \mu \mathrm{m})$ emission of PNe presented in this paper is based on these results. The first step consists in composing a set of template spectra of $\mathrm{PAH}^{0}, \mathrm{PAH}^{+}$, and VSGs. To do this we built an average spectrum of each specie from the spectra extracted by Rapacioli et al. and Berné et al. in NGC 7023, Ced 201, and the $\rho$-Ophiucus filament. The continuum from VSG spectra was removed since only bands are fitted (cf. Sect. 3.2). Each average spectrum is then fitted using a combination of Lorentzians and normalised so that

$$
\int_{6 \mu \mathrm{m}}^{14 \mu \mathrm{m}} I_{v}^{\mathrm{VSG}} \mathrm{d} v=\int_{6 \mu \mathrm{m}}^{14 \mu \mathrm{m}} I_{v}^{\mathrm{PAH}^{0}} \mathrm{~d} v=\int_{6 \mu \mathrm{m}}^{14 \mu \mathrm{m}} I_{v}^{\mathrm{PAH}^{+}} \mathrm{d} v=1
$$

where $I_{v}$ represents the specific intensity in the average spectra. The corresponding parameters are reported in Table A.1.

The basis consisting of the three PDR components was found to be insufficient for obtaining a satisfactory fit to the observations. Broad emission features $(\mathrm{BF})$ near 8 and $12 \mu \mathrm{m}$ are known to be prominent in post-AGB star spectra (Buss et al. 1993; Hrivnak et al. 2000; Kwok et al. 2001; Peeters et al. 2002) and a remnant of these bands could be present in PNe. Peeters et al. have classified as type $C$ the objects in which the $8.2 \mu \mathrm{m}$ $\mathrm{BF}$ is observed. We chose to use simple and necessarily approximated Gaussian profiles with centre at 8.2 and $12.3 \mu \mathrm{m}$ (cf. Table A.1) providing a reasonable fit to the bands observed in the post-AGB star IRAS 13416-6243, the prototype class C object from Peeters et al. (2002, cf. Fig. 2).

The "7.7 $\mu \mathrm{m}$ " band is known to be shifted near $7.8 \mu \mathrm{m}$ in PNe, whereas it occurs near $7.6 \mu \mathrm{m}$ in PDRs (cf. Table A.1). This means that a new PAH-type template spectrum has to be introduced into our fitting procedure. A redshifted band at $7.90 \mu \mathrm{m}$ was introduced with similar width and intensity compared to the

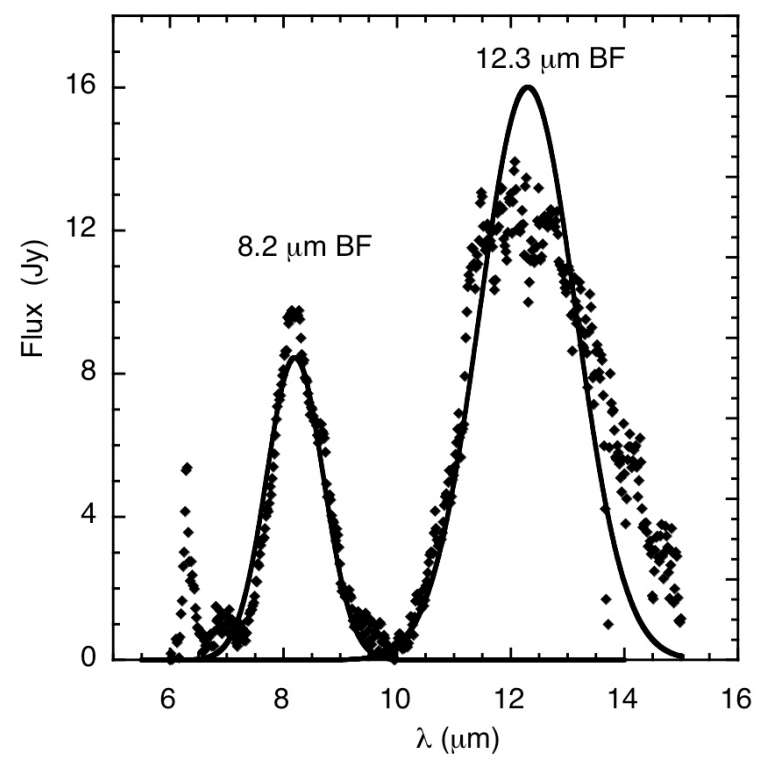

Fig. 2. Spectrum after continuum subtraction of the post-AGB star IRAS 13416-6243 (diamonds) defined as the type C class prototype by Peeters et al. (2002) and template spectra for the broad features (BF) at 8.2 and $12.3 \mu \mathrm{m}$ (full line), which are used in this work.

$7.64 \mu \mathrm{m}$ band of $\mathrm{PAH}^{+}$. A redshifted counterpart of the $8.6 \mu \mathrm{m}$ band was also identified $(8.65 \mu \mathrm{m}$ instead of $8.55 \mu \mathrm{m})$. No counterpart was found for the 6.2 and $11.2 \mu \mathrm{m}$ bands. We therefore used the characteristics of the $\mathrm{PAH}^{+}$bands at $6.28,11.20$, and $12.70 \mu \mathrm{m}$ to complete the new spectrum, which is assigned to a $\mathrm{PAH}^{x}$ population. To evaluate the impact of this assumption on the results of the fit, we built a second $\mathrm{PAH}^{x}$ spectrum with no bands in the 10-14 $\mu \mathrm{m}$ range. Possible candidates for the $\mathrm{PAH}^{x}$ population are discussed in Sect. 4.2.

\subsection{Fitting procedure}

In extreme irradiation conditions such as in PNe, grains at thermal equilibrium could also contribute to the mid-IR continuum. The description of this continuum would require detailed modelling for each source. To simplify, we only consider band emission. A continuum is therefore subtracted from the observations keeping an appropriate level for band wing emission. The continuum is made of linear slopes between 6.0 and $10 \mu \mathrm{m}$, and 10 and $14 \mu \mathrm{m}$. In the case of SWS spectra, the ionized gas lines are very narrow and can be easily subtracted. This is more complicated for the lower resolution spectra of IRS. However, only the [NeII] $12.8 \mu \mathrm{m}$ line is a concern for the fit because of possible blend with the $12.7 \mu \mathrm{m}$ PAH feature. A Gaussian profile line at $12.8 \mu \mathrm{m}$ was added to the basis for the fit. After continuum and line subtraction, the spectra are smoothed to the resolution of ISOCAM data $\lambda / \Delta \lambda=45$ (Boulanger et al. 2005). This is justified considering that the PDR components that enter the fit were extracted at that resolution (Rapacioli et al. 2005). This leads to a $F W H M$ of $0.3 \mu \mathrm{m}$ for the [NeII] line. The mid-IR band emission spectra of PNe is then projected on the basis defined by the six template spectra from Fig. 3 and the ionized gas line spectrum. The same approach was used for several compact H II regions. We define a Template Matrix $T$ which contains the basis spectra. The fit is realised by adjusting the parameters of a weight vector $w$ so that $\|v-w T\|^{2}$ is minimized, where $v$ is the observed band spectrum. This is achieved using the non-negative least squares (NNLS) minimization (Lawson \& Hanson 1974) which imposes $w>0$. 


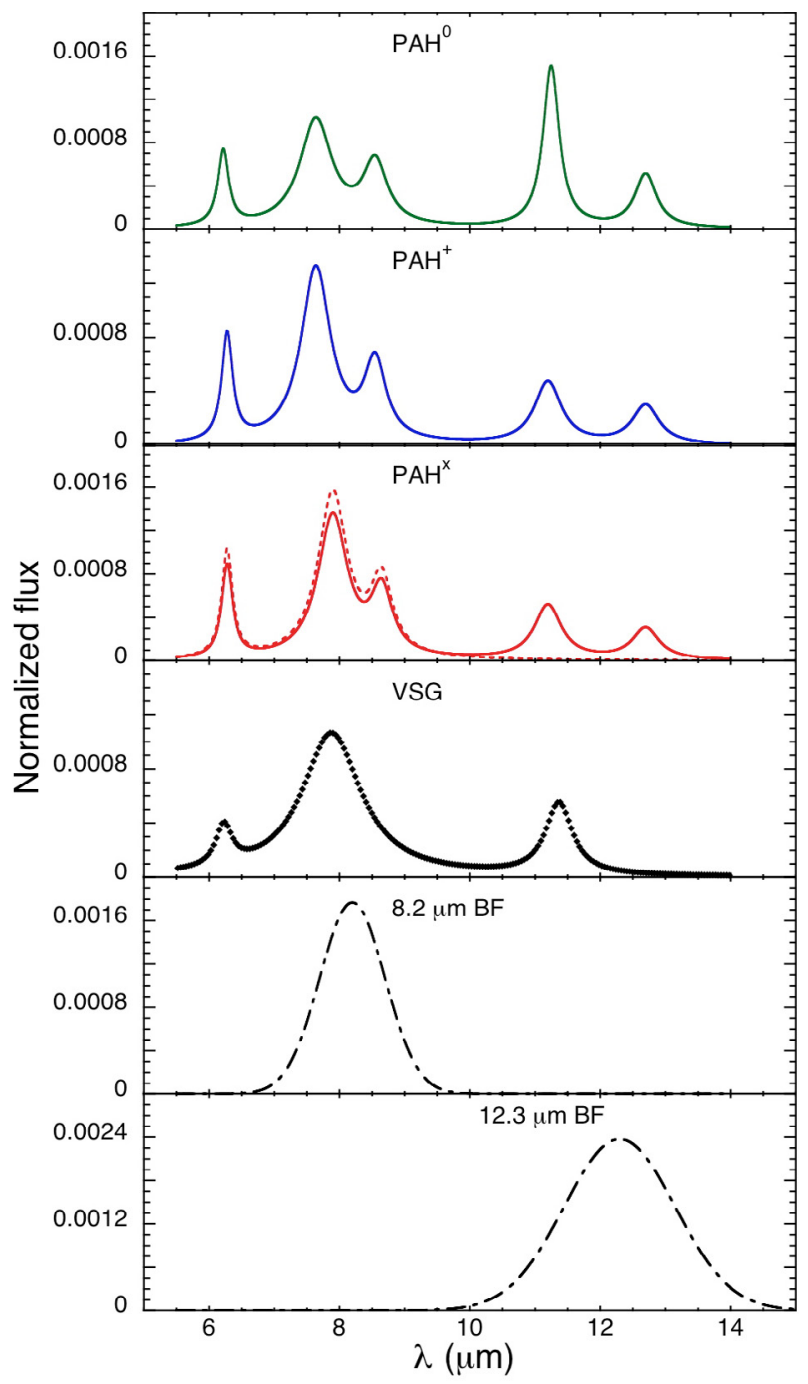

Fig. 3. Summary of the template spectra used in the fit of the midIR spectra. The $\mathrm{PAH}^{0}, \mathrm{PAH}^{+}$, and VSG components are adapted from Rapacioli et al. (2005); Berné et al. (2007). The $\mathrm{PAH}^{x}$ component is introduced in this paper (see Sect. 3.1) and has two possible associated spectra: one with bands in the 10-14 $\mu \mathrm{m}$ range (solid line), the other without (dashed line). The 8.2 and $12.3 \mu \mathrm{m} \mathrm{BFs} \mathrm{are} \mathrm{from} \mathrm{Fig.} \mathrm{2.} \mathrm{The}$ flux is normalized according to Eq. (1).

\subsection{Results}

This strategy physically interprets the nature of the mid-IR emitters in PNe. A fit of the data with spline or black body continuum and Lorentzian/Drude profiles (e.g. PAHFIT, Smith et al. 2007) will obviously give a better fit but will not give information on the properties of the emitters. Furthermore, our fit is linear and only has 6 free parameters ( 7 with the [NeII] line) ) so the adjustment for one observation is instantaneous. The results are presented in Figs. 4, 5 and Table A.2. The obtained fits appear quite reasonable with only one object (Hen 3-1333) over 18 making use of the 6 dust components. During the fitting procedure, we ran several tests. First, we found that the final results (e.g. the relative contribution of the different components) do not depend significantly on the exact definition of the continuum. Second, the dependence of the results on the assumption made for the $10-14 \mu \mathrm{m}$ spectrum of the $\mathrm{PAH}^{x}$ is given in Table A.2, showing that only slightly more $\mathrm{PAH}^{0}$ emission has to be included when the $\mathrm{PAH}^{x}$ do not emit in the $10-14 \mu \mathrm{m}$ range.
The $\mathrm{PAH}^{x}$ emission is found to be very high in galactic PNe, high in LMC/SMC PNe (with exception of SMP LMC 71), lower but still significant in galactic $\mathrm{H}$ II regions, and undetected in LMC H II regions. The $8.2 \mu \mathrm{m}$ BF is weak in all objects except in the PN Vo1. The BF features are in general weaker in galactic $\mathrm{H}$ II regions in which the spectra are clearly affected by extinction. To evaluate this effect, we added an extinction factor to the fit by using the cross-section provided by Weingartner \& Draine (2001a) for the molecular case $\left(R_{V}=5.5\right)$. The column density of matter carrying the extinction is a free parameter. We found that extinction is in the range $10-15 A_{V}$ in galactic $\mathrm{H}$ II regions, a few in LMC H II regions, and 1 at most in all PNe. Extinction effects are therefore significant only in the former objects. By including them, we obtained a strong increase in the $\mathrm{PAH}^{0}$ component and a decrease in the VSG component, which is consistent with extinction attenuating the $8.6 \mu \mathrm{m}$ and $11.3 \mu \mathrm{m}$ bands. This further decrease in the VSG component strengthens the fact that VSGs are found to be less abundant in galactic compared to LMC H II regions.

\section{Interpretation}

The analysis of the mid-IR emission performed in this work shows that there are additional mid-IR emitter components in PNe compared to the three components derived in PDRs by Rapacioli et al. (2005).

\subsection{Grain processing}

The first additional components are the carriers of the BFs at 8.2 and $12.3 \mu \mathrm{m}$, which could have associated continuum emission, but the second cannot be derived from our fitting procedure. These are very likely to be the remnants of the features observed in post-AGB stars (Hrivnak et al. 2000; Peeters et al. 2002) where the aromatic bands at 7.7 and $11.3 \mu \mathrm{m}$ are weak compared to the broad features at $\sim 8$ and $\sim 12 \mu \mathrm{m}$. There are also additional bands, in particular the $6.9 \mu \mathrm{m}$ aliphatic band (cf. Sect. 2 and Fig. 1 upper panels), suggesting that all these features are the signature of aliphatic material that is intimately connected to the aromatic material (Buss et al. 1993; Kwok et al. 2001; Goto et al. 2003; Sloan et al. 2007) and strongly evolves during the transition phase from AGB to PN. As the star becomes hotter, the material is processed by UV photochemistry. This could lead to larger aromatic systems (Kwok et al. 2001). Recent spatially resolved observations of the post-AGB object IRAS 22272+5435 (Goto et al. 2003) support a scenario of thermal processing that leads to an enrichment of aromatic versus aliphatic material. The results of our fits are consistent with this destruction scenario for the carriers of the BFs. For instance, the $8.2 \mu \mathrm{m} \mathrm{BF}$ is more intense in young PNe, the youngest object in our sample being PN Vo1 with $19 \%$ of the mid-IR band emission in this feature, whereas it is absent in the more evolved $\mathrm{PN} \mathrm{BD}+30^{\circ} 3639$. This band is also mainly absent in the studied LMC PNe and H II regions (cf. Figs. 4 and 5). In these cases, excitation effects may play a major role since only the hottest species are expected to emit at $8.2 \mu \mathrm{m}$. In particular, grains in LMC PNe may have moved farther away from the central object before ionization becomes significant as a consequence of a larger drift between dust and gas in lower gas density.

It has been shown in PDRs that PAHs are produced by destruction of VSGs at the surface of molecular clouds irradiated by UV photons (Rapacioli et al. 2005). VSGs are also present in PNe with a strong emission (up to $40-50 \%$ of the total midIR band intensity both in galactic and in LMC/SMC PNe). In 

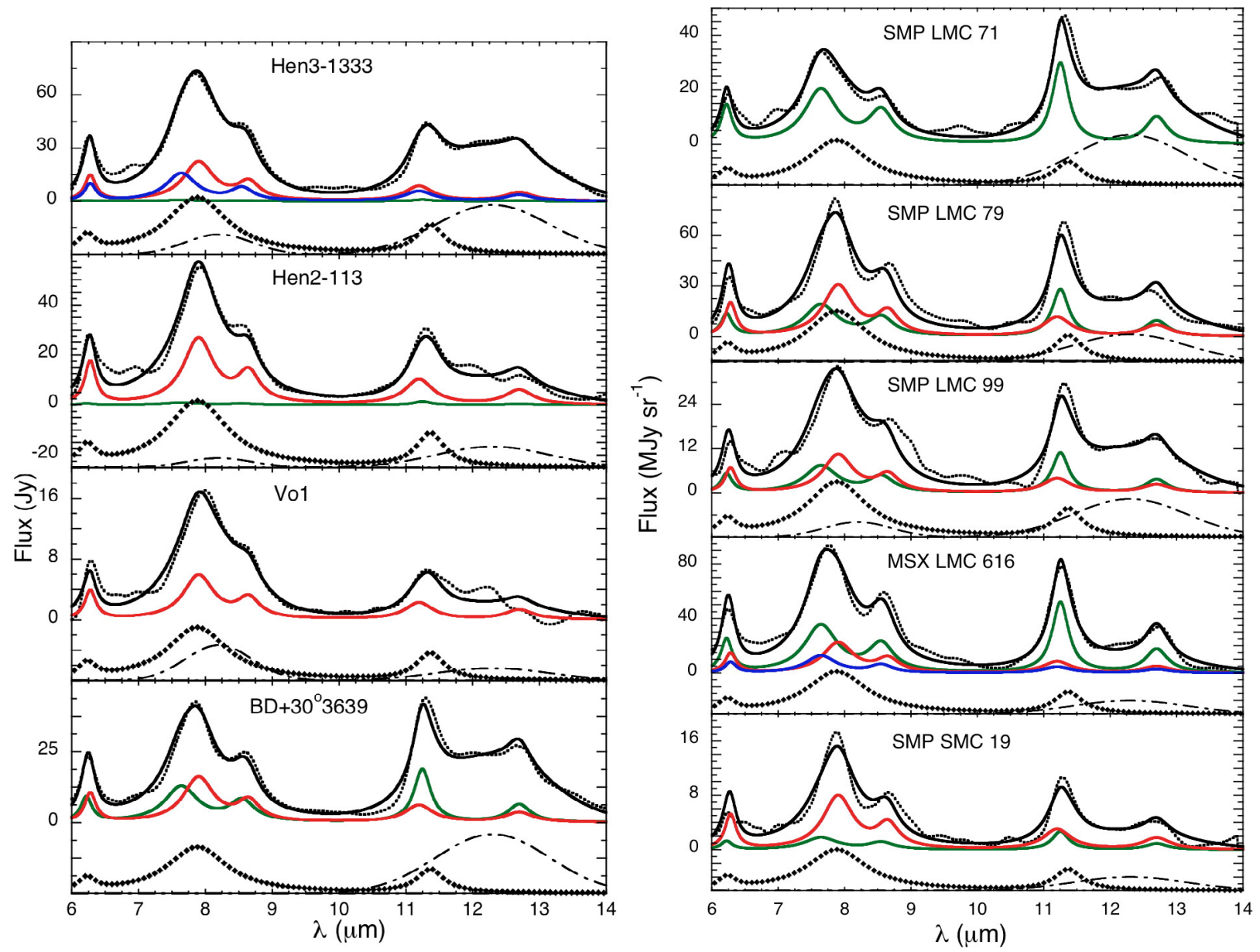

Fig. 4. PNe spectra after continuum subtraction and smoothing at the resolution of $\lambda / \Delta \lambda=45$ (dashed line) and fit using the template spectra displayed in Fig. 3 (solid line). The PAH components are displayed in colour lines: PAH neutrals (green), cations (blue), and PAH ${ }^{x}$ (red). The broader components have been shifted for clarity: VSGs (diamonds), 8.2, and $12.3 \mu \mathrm{m} \mathrm{BFs} \mathrm{(dash-dot} \mathrm{line).} \mathrm{The} \mathrm{ionic} \mathrm{gas} \mathrm{lines} \mathrm{have} \mathrm{been} \mathrm{removed}$ only in the galactic spectra recorded by SWS.

galactic H II regions, the VSG emission is significantly weaker (cf. Sect. 3.3). Clark et al. (1995) studied the emission in the 12 and $25 \mu \mathrm{m}$ IRAS bands in H II regions. They concluded that the $25 \mu \mathrm{m}$ carriers are bound conglomerates of basic structural units and that their destruction replenishes the $12 \mu \mathrm{m}$ carriers. This conclusion is in line with the results obtained by Rapacioli et al. in PDRs. That the 6-14 $\mu \mathrm{m}$ VSG emission is higher in LMC compared to galactic $\mathrm{H}$ II regions indicates that VSGs could survive longer in LMC, which reinforces the idea that these grains are localised farther away from the central object.

Lenzuni et al. (1989) underlined a dust-grain evolution scenario in PNe with an increase in the number of dust particles and a decrease in their size with the age of the object but the authors had difficulty concluding anything about the mechanisms driving this evolution. Both the carriers of the BFs and VSGs can be destroyed by far-UV photons in PNe and $\mathrm{H}$ II regions, and there is a trend toward decreasing abundance of these species with the age of the PN. The chemical relation between both types of grains, if any, is not clear though. Spatial information is required to validate an evolutionary scenario like the one observed for the PAH/VSG transition in PDRs.

\section{2. $P A H$ processing}

In our model, the $7.8 \mu \mathrm{m}$ band characteristic of Class $\mathrm{B}$ objects, essentially $\mathrm{PNe}$ and isolated Herbig AeBe stars (Peeters et al. 2002), is attributed to a PAH-type band located at $7.90 \mu \mathrm{m}$. Peeters et al. propose that the class B objects reveal pristine PAHs whereas in class A objects, which are typical of PDR-type environments, PAHs have been significantly processed by shock waves, UV photons, and cosmic rays. In this scenario, chemical pathways have to be found to reform the carriers of the $7.90 \mu \mathrm{m}$ band in protoplanetary disks where they are also found to strongly emit (Berné et al. 2008). However, as discussed in the following, our work suggests an opposite scenario in which the class B spectra reveal more processed PAH populations.

Candidates for the $7.90 \mu \mathrm{m}$ band and associated $\mathrm{PAH}^{x}$ spectrum were searched for in the on-line database of the spectral properties of PAHs from Malloci et al. (2007). We found that PAH anions might be plausible candidates since they have spectral characteristics with the right trend (Fig. 6): no shift for the $6.2 \mu \mathrm{m}$ band, redshift of the 7.7 and $8.6 \mu \mathrm{m}$ bands, and for the latter band this is better seen in the case of the large molecule $\mathrm{C}_{66} \mathrm{H}_{20}$. However, in the 10-14 $\mu \mathrm{m}$ range, the strong $\mathrm{CH}$ out-ofplane bending mode is significantly redshifted by about $0.5 \mu \mathrm{m}$ in anions compared to cations. Recent calculations on very large compact PAHs (Bauschlicher et al. 2008) show that this redshift tends to decrease with size. For instance, the main $\mathrm{CH}$ outof-plane bending band position was calculated to be 10.82 and $11.12 \mu \mathrm{m}$ for $\mathrm{C}_{130} \mathrm{H}_{28}^{+}$and $\mathrm{C}_{130} \mathrm{H}_{28}^{-}$, respectively. For comparison with the observations, the theoretical band positions have to be corrected for temperature effects, which generally lead to a 

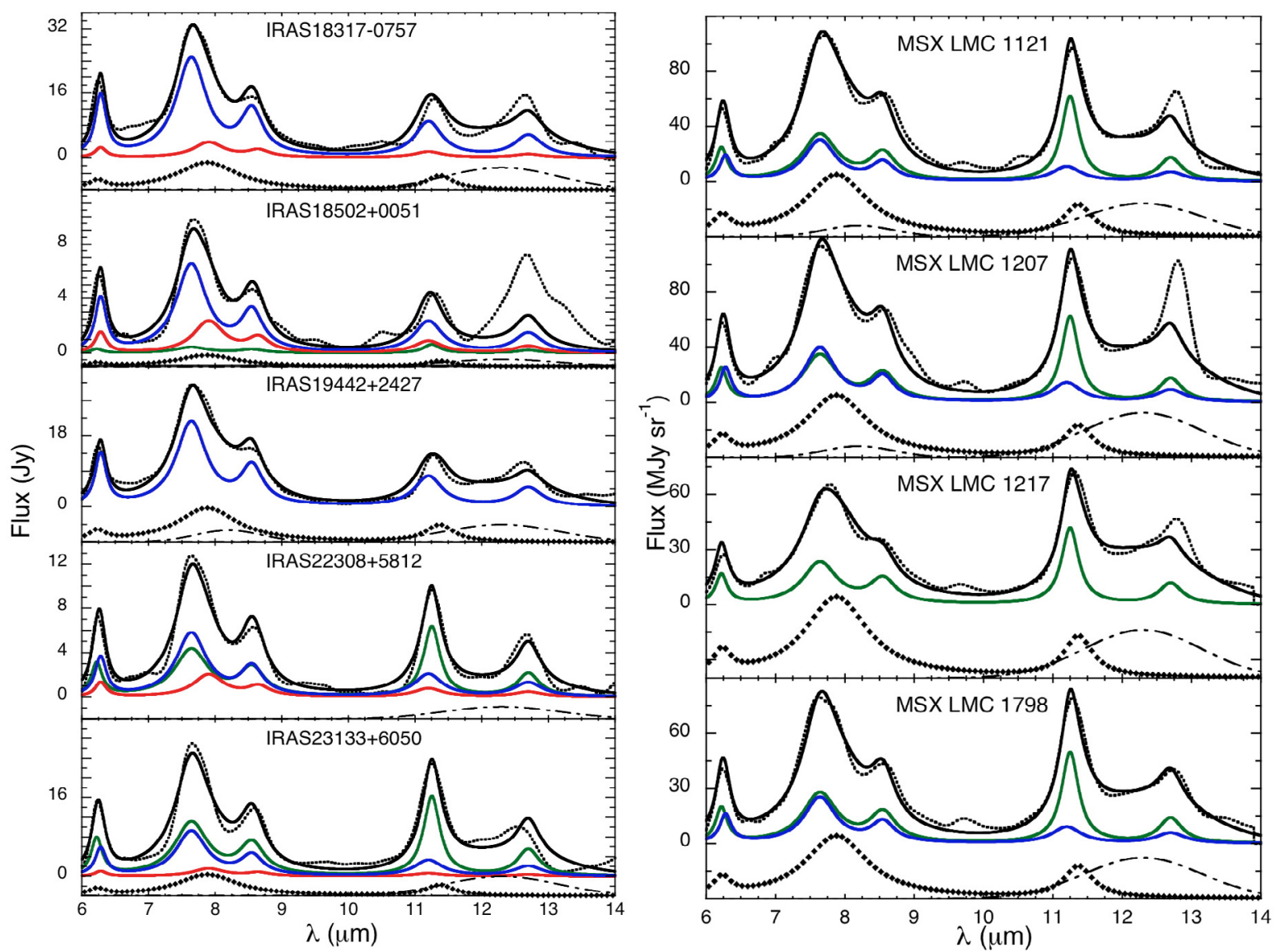

Fig. 5. Spectra of compact H II regions after continuum subtraction and smoothing at the resolution of $\lambda / \Delta \lambda=45$ (dashed line) and fit using the template spectra displayed in Fig. 3 (solid line). The PAH components are displayed in colour lines: PAH neutrals (green), cations (blue), and $\mathrm{PAH}^{x}$ (red). The broader components have been shifted for clarity: VSGs (diamonds), 8.2, and $12.3 \mu \mathrm{m}$ BFs (dash-dot line). The ionic gas lines have been removed only in the galactic spectra recorded by SWS. The [Ne II] line at $12.8 \mu \mathrm{m}$ has been introduced as an additional component in the IRS spectra in the LMC. It is not displayed on the plots.

redshift of the bands with temperature (Joblin et al. 1995). This effect has been quantified for only a few small and medium-sized neutral PAHs; therefore, we cannot decide whether large $\mathrm{PAH}^{-}$ or $\mathrm{PAH}^{+}$are better candidates for the $\mathrm{PAH}^{x}$ population. On the other hand, small and medium-sized $\mathrm{PAH}^{-}$are excluded since they would give a significant contribution in the $12 \mu \mathrm{m}$ range (cf. Fig. 6), which is not observed.

In their study of protoplanetary disks, Berné et al. (2008) conclude that $\mathrm{PAH}^{x}$ contain larger species than the PDR-type $\mathrm{PAH}^{0}$ and $\mathrm{PAH}^{+}$populations, since they are able to survive longer under the conditions of very high UV flux found in Herbig Ae stars. Furthermore, the emission of $\mathrm{PAH}^{x}$ in PDRs might be weaker due to milder excitation conditions, so its fingerprint might be lost in the intense $\mathrm{PAH}^{0} / \mathrm{PAH}^{+}$spectrum (cf. Fig. 7). PAH ${ }^{x}$ could absorb multiple UV photons when very close to the central star, which is clearly the case in protoplanetary disks (Berné et al. 2008). They could also absorb EUV photons $(h v>13.6 \mathrm{eV})$ in $\mathrm{H}$ II regions if they can survive from destruction. In the case of PNe, it is not excluded that some dust can survive in the ionized region as previously suggested by different authors (Natta \& Panagia 1981; Lenzuni et al. 1989; Stasińska \& Szczerba 1999; van Hoof et al. 2000). Ball et al. (1996) conclude from their study of ultra-compact H II regions that dust has to be located inside the ionized region or very close to it. Compiègne et al. (2007) measured PAH emission in the H II region facing the Horsehead nebula. Therefore the excitation of $\mathrm{PAH}^{x}$ by EUV photons is plausible.

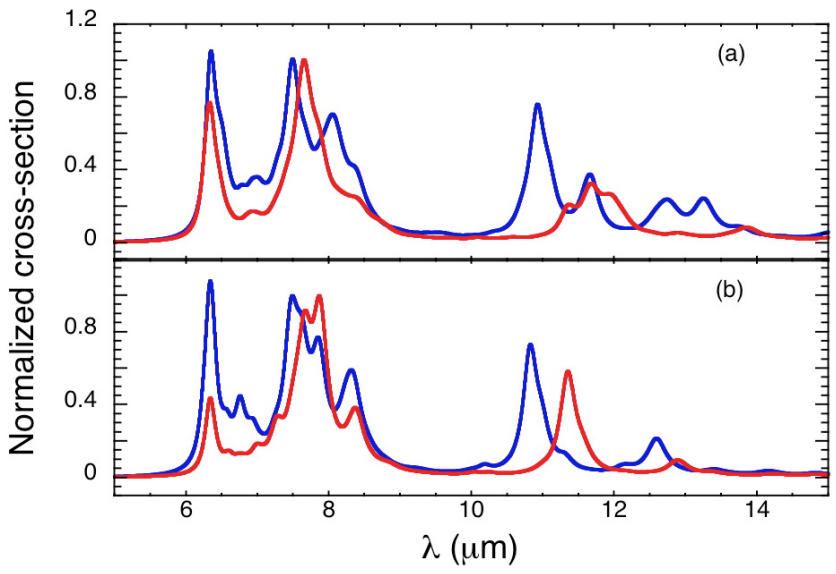

Fig. 6. Theoretical IR spectra from Malloci et al. (2007) with cations in blue and anions in red. a) Average of dibenzo[bc,kl]coronene $\left(\mathrm{C}_{30} \mathrm{H}_{14}\right)$, dibenzo[bc, ef]coronene $\left(\mathrm{C}_{30} \mathrm{H}_{14}\right)$, ovalene $\left(\mathrm{C}_{32} \mathrm{H}_{14}\right)$, dicoronylene $\left(\mathrm{C}_{48} \mathrm{H}_{20}\right)$, and circumovalene $\left(\mathrm{C}_{66} \mathrm{H}_{20}\right)$. b) Circumovalene $\left(\mathrm{C}_{66} \mathrm{H}_{20}\right)$ only. The spectra have been convolved with a Lorentzian line shape of $F W H M$ of 40 and $20 \mathrm{~cm}^{-1}$ for frequencies higher and smaller than $1000 \mathrm{~cm}^{-1}(10 \mu \mathrm{m})$, respectively. Normalization is to the maximum of the " 7.7 " $\mu \mathrm{m}$ feature.

Large anions, as well as cations, are good candidates for the $\mathrm{PAH}^{x}$ population from the point of view of spectroscopy. In terms of chemistry, the PAH anion scenario is attractive since 


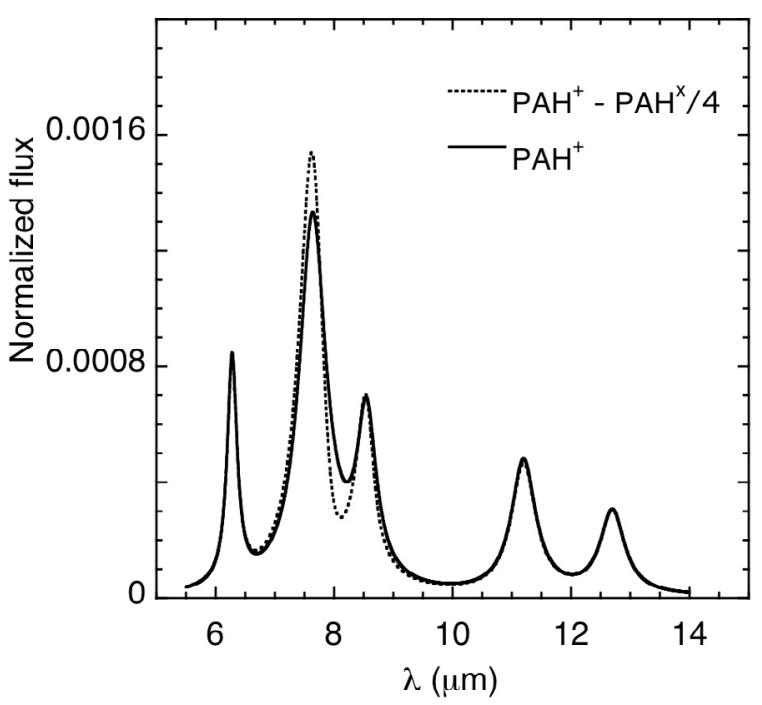

Fig. 7. Template $\mathrm{PAH}^{+}$spectrum (solid line) and $\mathrm{PAH}^{+}$spectrum after subtraction of a fraction of the $\mathrm{PAH}^{x}$ spectrum (1/4). The flux is normalized according to Eq. (1).

the electron density is high in PNe and attachment by collisions could overcome detachment by UV photons (cf. models by Bakes \& Tielens 1994; Weingartner \& Draine 2001b). Calculations of the PAH charge were performed for different values of the ionization parameter (Szczerba et al. 2008) and show that $\mathrm{PAH}^{-}$will be present in environments characterised by a small ionization parameter. In LMC/SMC PNe, the lower metallicity implies a lower dust density and therefore less attenuation of the UV field. Also, less dust implies a lower massloss rate during the AGB phase and consequently lower gas and electron densities in the PN phase. In terms of PAH charging, the combination of a higher UV flux and a lower value of $n_{\mathrm{e}}$ will not favour a large anion population. There is indeed a trend for the $\mathrm{PAH}^{x}$ component to be weaker in the LMC/SMC than in galactic PNe (cf. Fig. 4 and Table A.2). The extreme case is LMC 71 in which there is no evidence of $\mathrm{PAH}^{x}$. The properties of the LMC PNe are less known than those of galactic PNe. Still, Leisy \& Dennefeld (2006) assume for LMC 71 a value of $n_{\mathrm{e}}$ of $5000 \mathrm{~cm}^{-3}$, relatively higher than the values 3100, 3600, and $2300 \mathrm{~cm}^{-3}$ in LMC 79, LMC 99, and SMC 19, respectively. According to these authors, LMC 71 also has the highest excitation class: $T_{\text {eff }} \sim 80000 \mathrm{~K}$ and $\log L / L_{\odot}<4.27$ compared to $T_{\text {eff }} \sim 33000 \mathrm{~K}$ and $\log L / L_{\odot}<3.79$ in LMC 79 (Villaver et al. 2003). This could explain why $\mathrm{PAH}^{-}$cannot survive in LMC 71. For the galactic H II regions, the value of $n_{\mathrm{e}}$ is about 1500, 1200, 770, and $480 \mathrm{~cm}^{-3}$ in IRAS 18317-0757, IRAS 18502+0051, IRAS 22308+5812, and IRAS 23133+6050, respectively (Rudolph et al. 2006; Martín-Hernández et al. 2002). It is about two orders of magnitude lower than in PNe in which values in the range from about 50000 up to $100000 \mathrm{~cm}^{-3}$ have been determined for Hen 3-1333, Hen 2-113, and Vo1 (de Marco et al. 1997; Menzies \& Wolstencroft 1990), and a value of $21000 \mathrm{~cm}^{-3}$ in $\mathrm{BD}+30^{\circ} 3639$, the most evolved $\mathrm{PN}$ in our sample (Medina et al. 2006). A detailed modelling of the $\mathrm{PAH}$ charge in each of these regions is required, but this implies a good knowledge of the characteristics of each source.

\section{Conclusion}

The analysis of the mid-IR spectra in different objects has led to useful object classifications and some evolutionary scenario for PAH species (Hony et al. 2001; Peeters et al. 2002; van Diedenhoven et al. 2004; Sloan et al. 2007). However, these studies are limited by the difficulty interpreting the observed spectra as the sum of individual contributions tracing different chemical populations. The present analysis provides a more comprehensive view over the $6-14 \mu \mathrm{m}$ range based on a previous analysis of the mid-IR spectra of PDRs (Berné et al. 2007). We propose to rationalize the interpretation of the "7.7" $\mu \mathrm{m}$ feature in evolved stars with the following evolutionary scenario. In cool and less evolved stars (post-AGB stage), the emission is dominated by aliphatic rich material as suggested by different authors. In hotter star environments, this material has been processed and aromatic material dominates the emission. The destruction of VSGs to produce free PAHs is operating as observed in PDRs (Rapacioli et al. 2005; Berné et al. 2007), but there is an additional step in PAH processing that results in the selective emission of very large PAHs. The latter $\mathrm{PAH}^{x}$ population is only observed in extreme irradiation environments, most likely due to the combined effect of the destruction of the smallest PAHs with an increase in the temperature of $\mathrm{PAH}^{x}$ due to the high UV flux and the presence of EUV photons. Spatial information would be required, though, before concluding that the emitting $\mathrm{PAH}^{x}$ are located in H II regions, and this is indeed one of the goals of the SPECHII programme currently running on the Spitzer Space Telescope. Interestingly, the $\mathrm{PAH}^{x}$ species are also present in most LMC/SMC PNe, whereas they seem to be absent in LMC $\mathrm{H}$ II regions. This provides an observational diagnostic to differentiate both types of regions, which might be more reliable than the one usually used, e.g. the Buchanan et al. (2006) 2MASSMSX colour classification system (cf. for instance Kastner et al. 2008). Finally, a scenario in which these $\mathrm{PAH}^{x}$ are negatively charged is plausible but lack quantitative studies. If one could come to some conclusion on this point, then the $\mathrm{PAH}^{x}$ emission could be used to constrain the physical parameters (UV field, electronic density). Our goal is not to decide on this point but to lay the groundwork for more detailed studies, in particular involving a larger sample of objects and a better description of their physical parameters.

This work provides further support to the idea that evolved carbonaceous stars are the PAH nursery. The same scenario seems to apply in the $\mathrm{LMC} / \mathrm{SMC}$, indicating that the change in metallicity has not strongly affected the condensation sequence of aromatic dust, contrary to what has been suggested by some authors (Speck et al. 2006).

Acknowledgements. We thank the referee and the editor for useful comments that improved the manuscript. R.Sz. ackowledges support from grant N203 019 $31 / 2874$ of the Science and High Education Ministry of Poland. Support from the French national programme, Physique et Chimie du Milieu Interstellaire, is also acknowledged.

\section{References}

Bakes, E. L. O., \& Tielens, A. G. G. M. 1994, ApJ, 427, 822

Ball, R., Meixner, M. M., Keto, E., Arens, J. F., \& Jernigan, J. G. 1996, AJ, 112, 1645

Bauschlicher, Jr., C. W., Peeters, E., \& Allamandola, L. J. 2008, ApJ, 678, 316

Berné, O., Joblin, C., Deville, Y., et al. 2007, A\&A, 469, 575

Berné, O., Joblin, C., Fuente, A., \& Ménard, F. 2008, submitted

Boulanger, F., Lorente, R., Miville Deschênes, M. A., et al. 2005, A\&A, 436, 1151

Brandl, B. R., Bernard-Salas, J., Spoon, H. W. W., et al. 2006, ApJ, 653, 1129 Bregman, J. 1989, in Interstellar Dust, ed. L. J. Allamandola, \& A. G. G. M. Tielens, IAU Symp., 135, 109

Buchanan, C. L., Kastner, J. H., Forrest, W. J., et al. 2006, AJ, 132, 1890

Buss, Jr., R. H., Tielens, A. G. G. M., Cohen, M., et al. 1993, ApJ, 415, 250

Cernicharo, J., Heras, A. M., Tielens, A. G. G. M., et al. 2001, ApJ, 546, L123 
Cherchneff, I, Barker, J. R., \& Tielens, A. G. G. M. 1992, ApJ, 401, 269

Clark, F. O., Shipman, R. F., Assendorp, R., Kester, D., \& Egan, M. P. 1995, Planet. Space Sci., 43, 1353

Cohen, M., Tielens, A. G. G. M., Bregman, J., et al. 1989, ApJ, 341, 246

Compiègne, M., Abergel, A., Verstraete, L., et al. 2007, A\&A, 471, 205

de Marco, O., Barlow, M. J., \& Storey, P. J. 1997, MNRAS, 292, 86

Frenklach, M., \& Feigelson, E. D. 1989, ApJ, 341, 372

Goto, M., Gaessler, W., Hayano, Y., et al. 2003, ApJ, 589, 419

Hony, S., Van Kerckhoven, C., Peeters, E., et al. 2001, A\&A, 370, 1030

Hrivnak, B. J., Volk, K., \& Kwok, S. 2000, ApJ, 535, 275

Joblin, C., Boissel, P., Léger, A., D’Hendecourt, L., \& Défourneau, D. 1995, A\&A, 299, 835

Kastner, J. H., Thorndike, S. L., Romanczyk, P. A., et al. 2008, AJ, 136, 1221

Kwok, S., Volk, K., \& Bernath, P. 2001, ApJ, 554, L87

Lawson, C. L., \& Hanson, R. J. 1974, Solving least squares problems, PrenticeHall Series in Automatic Computation (Englewood Cliffs: Prentice-Hall)

Lebouteiller, V., Brandl, B., Bernard-Salas, J., Devost, D., \& Houck, J. R. 2007, ApJ, 665, 390

Leisy, P., \& Dennefeld, M. 2006, A\&A, 456, 451

Lenzuni, P., Natta, A., \& Panagia, N. 1989, ApJ, 345, 306

Malloci, G., Joblin, C., \& Mulas, G. 2007, Chem. Phys., 332, 353

Martín-Hernández, N. L., Peeters, E., Morisset, C., et al. 2002, A\&A, 381, 606

Medina, S., Pena, M., Morisset, C., \& Stasińska, G. 2006, Rev. Mex. Astron. Astrofis., 42, 53

Menzies, J. W., \& Wolstencroft, R. D. 1990, MNRAS, 247, 177

Natta, A., \& Panagia, N. 1981, ApJ, 248, 189

Peeters, E., Hony, S., Van Kerckhoven, C., et al. 2002, A\&A, 390, 1089
Peeters, E., Spoon, H. W. W., \& Tielens, A. G. G. M. 2004, ApJ, 613, 986

Rapacioli, M., Joblin, C., \& Boissel, P. 2005, A\&A, 429, 193

Reid, W. A., \& Parker, Q. A. 2006, MNRAS, 373, 521

Rudolph, A. L., Fich, M., Bell, G. R., et al. 2006, ApJS, 162, 346

Sloan, G. C., Jura, M., Duley, W. W., et al. 2007, ApJ, 664, 1144

Smith, J. D. T., Draine, B. T., Dale, D. A., et al. 2007, ApJ, 656, 770

Speck, A. K., Cami, J., Markwick-Kemper, C., et al. 2006, ApJ, 650, 892

Spoon, H. W. W., Marshall, J. A., Houck, J. R., et al. 2007, ApJ, 654, L49

Stanghellini, L., García-Lario, P., García-Hernández, D. A., et al. 2007, ApJ, 671,1669

Stasińska, G., \& Szczerba, R. 1999, A\&A, 352, 297

Szczerba, R., Omont, A., Volk, K., Cox, P., \& Kwok, S. 1997, A\&A, 317, 859

Szczerba, R., Górny, S. K., Stasińska, G., Siódmiak, N., \& Tylenda, R. 2001, Ap\&SS, 275, 113

Szczerba, R., Siódmiak, N., Stasińska, G., \& Borkowski, J. 2007, A\&A, 469, 799

Szczerba, R., Joblin, C., Berné, O., \& Szyszka, C. 2008, in Organic Matter in Space, ed. S. Kwok, et al. (Cambridge Univ. Press), IAU Symp., 251

Uchida, K. I., Sellgren, K., Werner, M. W., \& Houdashelt, M. L. 2000, ApJ, 530, 817

van Diedenhoven, B., Peeters, E., Van Kerckhoven, C., et al. 2004, ApJ, 611, 928 van Hoof, P. A. M., Van de Steene, G. C., Beintema, D. A., et al. 2000, ApJ, 532, 384

Villaver, E., Stanghellini, L., \& Shaw, R. A. 2003, ApJ, 597, 298

Weingartner, J. C., \& Draine, B. T. 2001a, ApJ, 548, 296

Weingartner, J. C., \& Draine, B. T. 2001b, ApJS, 134, 263 
C. Joblin et al.: Carriers of the mid-IR emission bands in PNe reanalysed, Online Material $p 1$

\section{Appendix A: Tables}

Table A.1. Positions, full widths at half maximum $(F W H M)$, and peak intensities (relative to the $7.7 \mu \mathrm{m}$ band) of the bands of the template spectra (cf. Fig. 3). Lorentzian shapes have been used except when specified. For $\mathrm{PAH}^{x}$, two cases are considered for the 10-14 $\mu \mathrm{m}$ range, one with bands similar to $\mathrm{PAH}^{+}$, the other in italics without bands.

\begin{tabular}{|c|c|c|c|c|c|c|c|c|c|c|}
\hline & \multicolumn{3}{|c|}{6.2} & & \multicolumn{3}{|c|}{7.7} & \multicolumn{3}{|c|}{8.6} \\
\hline & Pos. & $F W H M$ & Int. & & Pos. & $F W H M$ & Int. & Pos. & $F W H M$ & Int. \\
\hline $\mathrm{PAH}^{0}$ & 6.22 & 0.17 & 0.75 & & 7.64 & 0.60 & 1.0 & 8.55 & 0.45 & 0.57 \\
\hline $\mathrm{PAH}^{+}$ & 6.28 & 0.20 & 0.65 & & 7.64 & 0.55 & 1.0 & 8.55 & 0.40 & 0.45 \\
\hline \multirow{2}{*}{$\mathrm{PAH}^{x}$} & 6.28 & 0.20 & 0.65 & & 7.90 & 0.55 & 1.0 & 8.65 & 0.40 & 0.45 \\
\hline & 6.28 & 0.20 & 0.65 & & 7.90 & 0.55 & 1.0 & 8.65 & 0.40 & 0.45 \\
\hline VSG & 6.23 & 0.30 & 0.64 & & 7.88 & 1.10 & 1.0 & - & - & - \\
\hline \multirow[t]{3}{*}{$\dagger 8.2 \mu \mathrm{m} \mathrm{BF}$} & & & & & 8.20 & 1.18 & & & & \\
\hline & & 11.3 & & & & 12.7 & & & & \\
\hline & Pos. & $F W H M$ & Int. & & Pos. & $F W H M$ & Int. & & & \\
\hline $\mathrm{PAH}^{0}$ & 11.25 & 0.3 & 1.60 & & 12.70 & 0.4 & 0.54 & & & \\
\hline $\mathrm{PAH}^{+}$ & 11.20 & 0.5 & 0.38 & & 12.70 & 0.5 & 0.22 & & & \\
\hline PAH $^{x}$ & 11.20 & 0.5 & 0.38 & & 12.70 & 0.5 & 0.22 & & & \\
\hline & - & - & 0 & - & - & - & 0 & & & \\
\hline VSG & 11.37 & 0.5 & 0.32 & & & & & & & \\
\hline${ }^{\dagger} 12.3 \mu \mathrm{m} \mathrm{BF}$ & & & & & 12.30 & 2.00 & & & & \\
\hline
\end{tabular}

$\dagger$ Parameters for a Gaussian. 
C. Joblin et al.: Carriers of the mid-IR emission bands in PNe reanalysed, Online Material p 2

Table A.2. Integrated flux in the observed PNe for each component of the fit: $\mathrm{PAH}^{0}, \mathrm{PAH}^{+}, \mathrm{PAH}^{x}$, VSG, 8.2, and $12.3 \mu \mathrm{m}$ BFs (cf. spectra in Figs. 4 and 5). Numbers in parentheses show the relative contribution in $\%$ of the different ionization states of PAHs. For each object, the two templates of the $\mathrm{PAH}^{x}$ spectrum have been used (cf. Table A.1). [ArII] means a line at $6.99 \mu \mathrm{m},[\mathrm{ArIII}]$ at $8.99 \mu \mathrm{m},[\mathrm{SIV}]$ at $10.51 \mu \mathrm{m},[\mathrm{NeII}]$ at $12.81 \mu \mathrm{m}$, and $*$ means that the presence of the line emission was determined from the fitting procedure.

\begin{tabular}{|c|c|c|c|c|c|c|c|}
\hline \multirow[t]{2}{*}{ Object } & \multicolumn{6}{|c|}{$\begin{array}{l}\text { Normalized 6-14 } \mu \text { m integrated flux } \\
\text { (percentage of PAH flux) }\end{array}$} & \multirow[t]{2}{*}{$\begin{array}{l}\text { Ionized } \\
\text { gas lines }\end{array}$} \\
\hline & $\mathrm{PAH}^{0}$ & $\mathrm{PAH}^{+}$ & $\mathrm{PAH}^{x}$ & VSG & $8.2 \mu \mathrm{m} \mathrm{BF}$ & $12.3 \mu \mathrm{m} \mathrm{BF}$ & \\
\hline \multicolumn{8}{|l|}{ Galactic PNe } \\
\hline \multirow[t]{2}{*}{ Hen 3-1333 } & $0.01(0)$ & $0.15(42)$ & $0.21(58)$ & 0.38 & 0.09 & 0.16 & {$[\mathrm{NeII}]$} \\
\hline & $0.11(27)$ & $0.08(20)$ & $0.21(53)$ & 0.36 & 0.07 & 0.17 & \\
\hline \multirow{2}{*}{ Hen 2-113 } & $0.02(5)$ & 0 & $0.39(95)$ & 0.48 & 0.04 & 0.07 & weak [ArII], weak [ArIII], [NeII] \\
\hline & $0.13(33)$ & 0 & $0.26(67)$ & 0.47 & 0.05 & 0.09 & \\
\hline \multirow[t]{2}{*}{ Vo1 } & 0 & 0 & $0.31(100)$ & 0.45 & 0.19 & 0.05 & {$[\mathrm{NeII}]$} \\
\hline & $0.01(4)$ & 0 & $0.24(96)$ & 0.51 & 0.17 & 0.07 & \\
\hline \multirow[t]{2}{*}{$\mathrm{BD}+30^{\circ} 3639$} & $0.26(52)$ & 0 & $0.24(48)$ & 0.31 & 0 & 0.19 & [ArII], weak [ArIII], [NeII] \\
\hline & $0.33(65)$ & 0 & $0.18(35)$ & 0.29 & 0 & 0.20 & \\
\hline \multicolumn{8}{|l|}{ LMC/SMC PNe } \\
\hline \multirow[t]{2}{*}{ SMP LMC 71} & $0.46(100)$ & 0 & 0 & 0.35 & 0 & 0.19 & - \\
\hline & $0.46(100)$ & 0 & 0 & 0.35 & 0 & 0.19 & \\
\hline \multirow[t]{2}{*}{ SMP LMC 79} & $0.25(45)$ & 0 & $0.30(55)$ & 0.36 & 0 & 0.09 & - \\
\hline & $0.33(62)$ & 0 & $0.20(38)$ & 0.37 & 0 & 0.10 & \\
\hline \multirow[t]{2}{*}{ SMP LMC 99} & $0.20(48)$ & 0 & $0.22(52)$ & 0.38 & 0.07 & 0.13 & - \\
\hline & $0.27(69)$ & 0 & $0.12(31)$ & 0.40 & 0.07 & 0.14 & \\
\hline \multirow[t]{2}{*}{ MSX LMC 616} & $0.37(57)$ & $0.10(15)$ & $0.18(28)$ & 0.31 & 0 & 0.04 & weak [NeII]* \\
\hline & $0.44(69)$ & $0.06(9)$ & $0.14(22)$ & 0.31 & 0 & 0.05 & \\
\hline \multirow[t]{2}{*}{ SMP SMC 19} & $0.13(24)$ & 0 & $0.42(76)$ & 0.39 & 0 & 0.06 & weak $[\mathrm{NeII}]^{*}$ \\
\hline & $0.25(46)$ & 0 & $0.29(54)$ & 0.38 & 0 & 0.08 & \\
\hline \multicolumn{8}{|l|}{ Galactic H II regions } \\
\hline \multirow[t]{2}{*}{ IRAS18317-0757 } & 0 & $0.62(87)$ & $0.09(13)$ & 0.21 & 0 & 0.08 & {$[$ ArII $],[$ ArIII $]$, weak [SIV], [NeII] } \\
\hline & 0 & $0.62(85)$ & $0.11(15)$ & 0.18 & 0 & 0.09 & \\
\hline \multirow[t]{2}{*}{ IRAS18502+0051 } & $0.05(6)$ & $0.61(70)$ & $0.21(24)$ & 0.10 & 0 & 0.03 & [ArII], [ArIII], weak [SIV], [NeII] \\
\hline & $0.14(16)$ & $0.55(62)$ & $0.19(22)$ & 0.07 & 0 & 0.05 & \\
\hline \multirow[t]{2}{*}{ IRAS19442+2427 } & 0 & $0.58(100)$ & 0 & 0.29 & 0.06 & 0.07 & {$[\mathrm{ArII}],[\mathrm{NeII}]$} \\
\hline & 0 & $0.56(89)$ & $0.07(11)$ & 0.26 & 0.04 & 0.07 & \\
\hline \multirow[t]{2}{*}{ IRAS22308+5812 } & $0.38(42)$ & $0.39(43)$ & $0.13(15)$ & 0.06 & 0 & 0.04 & [ArII], [ArIII], [NeII] \\
\hline & $0.44(48)$ & $0.35(39)$ & $0.12(13)$ & 0.04 & 0 & 0.05 & \\
\hline \multirow[t]{2}{*}{ IRAS23133+6050 } & $0.44(57)$ & $0.28(36)$ & $0.05(7)$ & 0.16 & 0 & 0.07 & [ArII], [ArIII], weak [SIV], [NeII] \\
\hline & $0.45(59)$ & $0.28(37)$ & $0.03(4)$ & 0.17 & 0 & 0.07 & \\
\hline \multicolumn{8}{|l|}{ LMC H II regions } \\
\hline MSX LMC 1121 & $0.31(61)$ & $0.20(39)$ & 0 & 0.36 & 0.04 & 0.09 & {$[\mathrm{NeII}]^{*}$} \\
\hline MSX LMC 1207 & $0.28(54)$ & $0.24(46)$ & 0 & 0.33 & 0.04 & 0.11 & {$[\mathrm{NeII}] *$} \\
\hline MSX LMC 1217 & $0.31(100)$ & 0 & 0 & 0.54 & 0 & 0.15 & {$[\mathrm{NeII}]^{*}$} \\
\hline MSX LMC 1798 & $0.32(60)$ & $0.21(40)$ & 0 & 0.36 & 0 & 0.11 & - \\
\hline
\end{tabular}

